\title{
Le développement de l'apprentissage dans le supérieur : une évaluation empirique à partir de l'enquête Génération 2001
}

The development of apprenticeship in higher education: an empirical evaluation from the "Generation 2001" survey

\section{Sabina Issehnane}

\section{OpenEdition}

\section{Journals}

Édition électronique

URL : http://journals.openedition.org/travailemploi/4946

DOI : 10.4000/travailemploi.4946

ISSN : 1775-416X

Éditeur

DARES - Ministère du Travail

Édition imprimée

Date de publication : 15 mars 2011

Pagination : 27-39

ISSN : 0224-4365

\section{Référence électronique}

Sabina Issehnane, «Le développement de l'apprentissage dans le supérieur : une évaluation empirique à partir de l'enquête Génération 2001 », Travail et Emploi [En ligne], 125 | janvier-mars 2011, mis en ligne le 14 janvier 2013, consulté le 19 avril 2019. URL : http://journals.openedition.org/travailemploi/4946 ; DOI : 10.4000/travailemploi.4946 


\title{
Le développement de l'apprentissage dans le supérieur : une évaluation empirique à partir de l'enquête Génération 2001
}

Sabina Issehnane $\left(^{*}\right)$

\begin{abstract}
On constate ces dernières années une élévation du niveau des diplômes concernés par l'apprentissage. L'objectif de cet article est d'étudier si les apprentis, qu'ils soient du secondaire ou surtout du supérieur, ont une meilleure insertion professionnelle, à savoir un accès à l'emploi plus probable et un salaire plus élevé, que ceux qui sont passés par la voie scolaire standard, autrement dit ceux qui ont effectué le même type d'études mais sans passer par l'apprentissage. L'auteure utilise pour cela une évaluation microéconométrique, à partir de l'enquête Génération 2001 du Céreq. Les résultats montrent que, après avoir corrigé du caractère non aléatoire de l'apprentissage, l'impact de l'apprentissage sur l'emploi devient non significatif pour les sortants du supérieur, alors que sans correction, il était positif. Pour le secondaire, l'effet sur l'emploi reste significatif et positif. L'apprentissage a un effet positif sur le salaire pour les deux populations.
\end{abstract}

Le contrat d'apprentissage est le contrat aidé qui connait la plus grande progression en termes d'effectifs et cette évolution devrait durer compte tenu des orientations actuelles. La loi de programmation pour la Cohésion sociale du 18 janvier 2005 a fixé comme objectif que le nombre d'apprentis atteigne 500000 personnes en 2009. Cet objectif a presque été atteint, la Dares(1) estime en effet à 413000 le nombre d'apprentis en France en 2009, alors qu'ils étaient près de 200000 au début des années 1990 . Cette croissance s'est accompagnée d'une élévation des niveaux de formation concernés par l'apprentissage (Arrighi, Brochier, 2005a). En effet, on constate ces dernières années que l'apprentissage est davantage présent dans l'enseignement supérieur (SANCHEZ, 2007 et 2010). À titre d'exemple, les formations de niveaux $\mathrm{Bac}+3$ et plus reçoivent 10000 nouveaux apprentis de plus chaque année. En 2008, $21 \%$ des nouveaux contrats visent l'obtention d'un diplôme de l'enseignement supérieur, contre $13 \%$ en 2005 , et $6 \%$ dix ans plus tôt (ibid.). Cette tendance est accentuée par la volonté des pouvoirs publics de développer la professionnalisation des études à tous les niveaux. Ainsi, on peut s'interroger sur le développement récent de l'apprentissage au sein de l'enseignement supérieur, afin d'apprécier si cette évolution est vraiment bénéfique, au regard notamment de l'apprentissage dans le secondaire.
L'apprentissage s'est développé initialement pour permettre aux jeunes pas ou peu diplômés d'accéder à une formation tout en leur donnant une première expérience professionnelle. Ce dispositif est devenu un instrument important de la politique de l'emploi afin de lutter contre le chômage des jeunes. Il constitue à la fois un mode d'acquisition d'une qualification et une mesure publique de l'emploi. Il a pour but de fournir aux jeunes une qualification professionnelle sanctionnée par un diplôme. Il consiste en une formation générale, théorique et pratique qui se déroule en alternance avec à la fois des enseignements théoriques en centre de formation d'apprentis (CFA) et des enseignements du métier en entreprise. Ce dispositif d'alternance, en cherchant à faciliter la transition entre la sortie du système éducatif et l'accès à un emploi stable, constitue un sas entre la formation et l'emploi. La formation initiale étant jugée, en France, trop éloignée du monde du travail, les pouvoirs publics ont cherché à développer des formations professionnelles également au sein de l'enseignement supérieur (HeTzel, 2006; LunEL, 2007). Cette tendance s'inscrit notamment dans la réforme $\operatorname{LMD}(2)$, qui vise à introduire une séquence qui soit professionnalisante en fin de licence. Le développement actuel de la professionnalisation des filières de l'enseignement supérieur, ainsi que le montant des dépenses engagées pour ces mesures, conduisent à s'interroger réellement quant à l'efficacité de l'apprentissage, relativement

(2) Du nom des trois diplômes Licence, Master et Doctorat. Cette réforme s'inscrit dans le processus de Bologne (1999) qui vise à créer un «modèle européen de l'enseignement supérieur».
(*) CEPN - Université Paris 13 et Centre d'Études de l'Emplo (CEE); sabina.issehnane@cee-recherche.fr

(1) Direction de l'animation de la recherche, des études et des statistiques du Ministère en charge du travail et de l'emploi. 
aux formations traditionnelles en ce qui concerne l'insertion professionnelle des jeunes.

Cette tendance pourrait se justifier par une insertion des jeunes qui reste difficile, y compris pour les diplômés du supérieur, bien que le diplôme constitue une protection contre le chômage en France. En étudiant les primo-sortants (3) de la «Génération $2001 »$, on observe que ces derniers connaissent des conditions d'insertion sur le marché du travail plus difficiles que leurs prédécesseurs. Ils sortent de formation initiale en 2001, certes dans un contexte assez favorable, mais la conjoncture économique se dégrade peu après, ce qui rend difficile l'insertion professionnelle des jeunes non immédiatement embauchés, ainsi que leur stabilisation sur le marché du travail (Giret, Molinari-Perrier, Moullet, 2006). Si on considère les jeunes sortis de l'enseignement supérieur, le taux de chômage en 2001 s'élève à $12 \%$, il descend à $8 \%$ un an plus tard mais remonte à $11 \%$ en 2004 , suite à la dégradation de la conjoncture économique, soit presque au même niveau qu'à leur entrée sur le marché du travail. En 2001, il correspond au double du taux de chômage des jeunes sortis de l'enseignement supérieur en 1998, qui ont bénéficié d'une conjoncture plus favorable. Ce taux est néanmoins bien inférieur à celui de l'ensemble de la génération 2001, qui se situe autour des $16 \%$. Trois ans après être sortis de formation initiale, le taux de chômage des jeunes non diplômés s'élève à $39 \%$. L'insertion professionnelle diffère ainsi fortement selon le niveau de formation initiale. Bien qu'étant dans une situation moins difficile que les jeunes moins diplômés, les sortants du supérieur connaissent également des difficultés d'insertion.

Cette étude consiste en une analyse multidimensionnelle de l'efficacité de l'apprentissage sur la base de deux critères : la probabilité d'être en emploi un an après être sorti du dispositif et le salaire alors perçu. L'analyse porte sur les sortants de l'enseignement supérieur mais nous étudions également les sortants du secondaire comme éléments de comparaison. Nous mobilisons pour cela les données de l'Enquête Génération 2001 du Céreq. Cet article fait l'objet de cinq sections. La première examine la croissance de l'apprentissage en France ces dernières années, notamment dans les formations du supérieur, et la deuxième expose les travaux antérieurs portant sur l'évaluation de l'apprentissage. La troisième section s'attache à présenter les données que nous mobilisons. La méthode d'estimation utilisée est exposée dans la section suivante. Enfin, la dernière section fournit les résultats de l'évaluation quantitative de l'effet de l'apprentissage sur l'insertion professionnelle des bénéficiaires.

(3) On appelle primo-sortant tout jeune ayant quitté le système éducatif pour la première fois et pour une durée minimale d'un an.

\section{L'évolution de l'apprentissage en France}

Le nombre d'apprentis en France a fortement augmenté depuis 1995 (de l'ordre de 130000 nouveaux apprentis). Après une stabilisation au début des années 2000, les effectifs sont repartis largement à la hausse depuis pour atteindre désormais 427650 apprentis en 2009(4). L'apprentissage a changé de visage (Durier, SAING, 2007). Il n'est plus réservé qu'aux élèves du secondaire professionnel, mais participe à la formation désormais de milliers d'étudiants de l'enseignement supérieur, du BTS aux écoles d'ingénieur en passant par les Licences et les Masters universitaires. L'augmentation du poids de l'enseignement supérieur dans l'apprentissage est visible dans le graphique 1 qui retrace l'évolution depuis plus de trente ans des effectifs des différents niveaux de diplômes préparés en apprentissage. Les effectifs de niveau $\mathrm{V}(5)$ sont restés à peu près constants depuis le début des années 1980 (autour des 230000), tandis que les autres niveaux de diplôme ont vu leurs effectifs nettement augmenter à partir du milieu des années 1990 (6). Les effectifs dans l'enseignement supérieur (niveaux I à III) ont quadruplé entre 1995 et 2009. En 2009, les apprentis préparant un diplôme de niveaux I à III sont plus de 100000 en France. Ils représentent désormais plus de $20 \%$ de l'effectif total des apprentis.

\section{Graphique 1 : Évolution des effectifs d'apprentis en fonction du diplôme préparé}

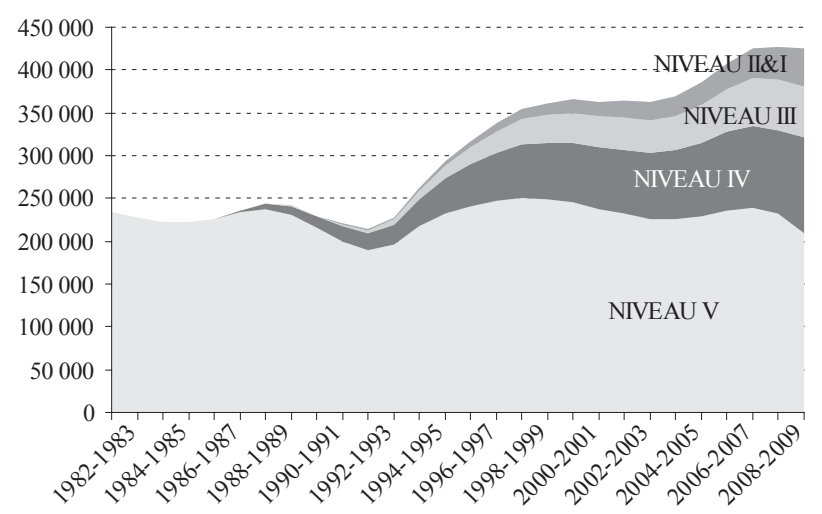

Source : Calculs DEPP./Men à partir des enquêtes sur les effectifs des centres de formation d'apprentis (enquête 51).

Plusieurs raisons peuvent expliquer cette évolution. D'un côté, les écoles et les universités peuvent être poussées à proposer des formations en apprentissage afin d'attirer des étudiants soucieux, dans un climat concurrentiel, d'acquérir une expérience

(4) D'après les données de la Direction de l'évaluation, de la prospective et de la performance (Depp) du Ministère de l'Éducation nationale.

(5) Sortis de deuxième année de CAP / BEP avec ou sans diplôme, ou de seconde ou de première.

(6) À partir de 1985, on voit apparaître la montée du niveau IV qui correspond à la création des baccalauréats professionnels. 
professionnelle. Les étudiants y recherchent peutêtre également la possibilité d'obtenir un revenu et, pour ceux qui font le choix d'une école, d'être exonérés des frais de scolarité. Il permet en outre d'être encadré par un contrat de travail, ce que l'alternative du stage ne permet pas. L'étude de Prisca KergoAt (2010) montre que la première hypothèse est davantage vérifiée. Ce sont avant tout les étudiants de milieux favorisés qui font le choix de l'apprentissage afin d'améliorer leurs chances d'insertion professionnelle. Leur objectif est d'obtenir «un avantage concurrentiel» en acquérant une expérience pratique en entreprise, qu'ils espèrent davantage valorisable sur le marché du travail à la sortie de leur formation initiale. Ces motivations rendent les formations par apprentissage plus sélectives car elles attirent davantage d'étudiants. En outre, l'apprentissage n'est pas synonyme de démocratisation scolaire. D'une part, les apprentis du supérieur sont issus des mêmes milieux (favorisés) que les autres étudiants et, d'autre part, «seuls $12 \%$ des apprentis du supérieur sont issus d'une formation par apprentissage» (KERGOAT, 2010). L'apprentissage ne permet pas aux jeunes les plus défavorisés d'obtenir un niveau de diplôme plus élevé, il aurait même tendance à renforcer les inégalités existantes. Ces éléments invitent à questionner les caractéristiques individuelles des apprentis, notamment ceux du supérieur, et à l'effet propre de l'apprentissage sur l'insertion professionnelle.

\section{L'apprentissage en question}

Une importante littérature théorique s'est développée sur les motivations des entreprises à investir dans le capital humain (Acemoglu, PischKe, 1999), et spécifiquement dans l'apprentissage (LindLeY, 1975; ACEMoglu, PischKe, 1998; Fougère, SCHWERDT, 2002). Deux principaux arguments sont mis en avant. Le premier est de réduire le coût du travail. Le deuxième tient dans l'idée que les entreprises veulent investir dans ce type de «capital», soit parce qu'elles font face à une pénurie de maind'œuvre qualifiée, soit parce qu'elles envisagent de former directement pour ensuite embaucher. Stefan BENDER et Wolfgang SCHWERDT (2000) montrent que le premier argument prévaut en France. Autrement dit, l'utilisation de l'apprentissage par les entreprises est d'abord motivée par le faible coût du travail des apprentis. En France, les pouvoirs publics participent activement à cet investissement (MAINAUD, 2007). L'employeur bénéficie d'exonérations de cotisations patronales et salariales, d'un crédit d'impôt apprentissage, ainsi que d'une indemnité compensatrice forfaitaire. Le jeune, quant à lui, est rémunéré en pourcentage du SMIC en fonction de son âge et de sa progression dans le cycle de la formation objet de l'apprentissage. Le salaire minimum des apprentis varie ainsi de $25 \%$ à $78 \%$ du SMIC.

\section{Encadré 1}

\section{L'organisation de l'apprentissage dans le supérieur}

Au milieu des années 1990, on voit apparaître le développement d'un modèle spécifique d'organisation de l'apprentissage dans le supérieur. Les universités, à l'instar des lycées qui préparent des BEP en apprentissage, peuvent assurer la responsabilité pédagogique des formations en apprentissage et délivrer les diplômes qui y sont associés. Ces derniers bénéficient d'une double habilitation, du Ministère de l'Enseignement supérieur (et de la Recherche) et de la Région. Afin de créer une Unité de formation en apprentissage (UFA), les universités concluent une convention avec un Centre de formation d'apprentis (CFA). Des CFA ont ainsi été créés pour les formations du supérieur autres que le BTS. Les formations effectuées en apprentissage se déroulent dans les locaux universitaires. En revanche, même si l'organisme gestionnaire peut être l'université, les CFA sont souvent «hors-murs » et la gestion est alors assurée par un organisme externe (souvent une association), qui peut gérer plusieurs CFA. Le rôle de ces organismes est avant tout administratif, juridique et financier. Les Conseils régionaux vont pousser les universités situées sur un même territoire régional à se regrouper au sein de ces entités, en associant non seulement les universités mais également les représentants des branches professionnelles, avec pour objectif d'adapter en permanence les formations aux besoins des entreprises et des régions. Ces organismes reçoivent la majorité de leur financement de la taxe d'apprentissage et perçoivent également des versements de la part du Conseil régional et des OPCA(7). La taxe d'apprentissage (8) (loi du 16 juillet 1971) est un impôt obligatoire auquel les entreprises sont assujetties(9) chaque année afin de soutenir financièrement les formations initiales des enseignements technologiques et professionnels. C'est le seul impôt pour lequel les entreprises ont la liberté de choisir le ou les bénéficiaires du versement.

L'évaluation des dispositifs des politiques de l'emploi et de la formation professionnelle a fait l'objet de nombreux travaux (Bonnal, FougÈre, Serandon, 1997; Fougère, Kramarz, Magnac, 2000; Brodaty, Crépon, Fougère, 2007; Even, KLEIN, 2006). Les travaux français centrés sur l'apprentissage montrent que ce dispositif réduit la probabilité d'être au chômage mais a des effets faibles ou nuls sur les salaires post-apprentissage

(7) Les OPCA (Organismes paritaires collecteurs agréés) ont pour fonction de collecter les versements effectués par les entreprises afin de financer les dispositifs de formation professionnelle.

(8) Le taux de la taxe d'apprentissage globale est de $0,68 \%$ de la masse salariale.

(9) Sont exonérées du paiement de cette taxe les entreprises dont le but exclusif est la formation première et les entreprises ayant employé un ou plusieurs apprentis pendant l'année et dont la masse salariale brute n'excède pas 95513 euros annuel. 
(Sollogoub, Ulrich, 1999; Simmonet, Ulrich, 2000; Fougère, Goux, Maurin, 2001; Aubriac, Rathelot, Sanchez, 2009). Michel Sollogoub et Valérie UlRICH (1999) comparent l'apprentissage avec le lycée professionnel. Ils montrent que l'apprentissage réduit de façon significative la probabilité d'être au chômage mais a peu d'effets sur les salaires postérieurs. On peut considérer que les apprentis, usuellement appelés «faux débutants » lorsqu'ils accèdent au marché du travail, du fait de l'expérience professionnelle acquise lors de leur formation initiale, sont «plus employables», si bien qu'ils bénéficieraient d'atouts sur le marché du travail que les autres passés par la voie scolaire habituelle n'auraient pas. Véronique SIMMONET et Valérie ULRICH (2000) montrent, à partir de l'enquête Jeunes et Carrières de l'Insee, que les apprentis ont une plus grande probabilité d'être en emploi que les jeunes ayant suivi un cursus dans un lycée professionnel, et une probabilité moindre de travailler à temps partiel. Cependant, les auteurs ne constatent pas de différences au niveau des salaires (d'embauche et croissance de salaire). Denis FougĖre, Dominique Goux et Eric MaURIN (2001) trouvent également que l'apprentissage n'a pas d'effet spécifique sur les salaires post-apprentissage. D'autres études montrent que, trois ans après la sortie de la formation initiale, les jeunes ayant effectué leurs études par apprentissage sont plus souvent en emploi que ceux qui ont suivi leur cursus par la voie scolaire. Néanmoins, ce différentiel décroît quand le niveau de formation augmente (ARRIGHI, JosePH, 2005). La proportion d'apprentis qui sont en emploi trois ans après être sortis du système éducatif varie fortement selon les spécialités.

Ces différentes études se sont principalement intéressées à la comparaison entre les sortants de lycées professionnels et ceux ayant effectué le même type de formation mais par apprentissage. Elles portent ainsi sur les formations professionnelles qui ont intrinsèquement vocation à dispenser une formation pratique en entreprise aux côtés de la formation théorique. Cette expérience en entreprise a lieu soit par le biais d'un stage, soit par le biais de l'apprentissage. À notre connaissance, il n'existe pas d'étude évaluant l'apprentissage dans le supérieur. Ainsi, nous proposons d'évaluer, d'une part les effets de l'apprentissage dans le secondaire, et d'autre part dans l'enseignement supérieur, afin d'étudier la pertinence du développement de ces dispositifs dans le supérieur.

\section{Données et éléments descriptifs}

Afin d'estimer l'efficacité du contrat d'apprentissage pour les primo-sortants de formation initiale, nous utilisons l'enquête Génération 2001. Nous présentons tout d'abord les données puis quelques éléments descriptifs du profil des apprentis.

\section{Les données}

L'Enquête Génération 2001 du Céreq est une enquête longitudinale portant sur les premières années de vie active des jeunes sortis de formation initiale en 2001. Elle comprend un calendrier décrivant mois par mois la situation des jeunes. Elle permet ainsi d'analyser les trajectoires d'entrée dans la vie active et les transitions de la formation vers l'emploi. En plus du parcours professionnel, l'enquête a l'avantage de rassembler diverses données sur les individus : caractéristiques sociodémographiques, informations à caractère subjectif. Deux enquêtes ont déjà eu lieu auprès des jeunes sortis de formation initiale en 1992 et 1998. Contrairement aux deux autres, l'enquête de 2001 n'a fait l'objet que d'une seule interrogation en 2004, trois ans après la sortie du système éducatif. Au cours de cette enquête, le Céreq a interrogé un échantillon de 10000 jeunes parmi les 760000 jeunes sortis de formation initiale en 2001. Cette enquête a fait l'objet d'extensions réalisées à la demande des régions et des Ministères : 15000 individus supplémentaires ont été interrogés.

Les données de l'Enquête Génération 2001 sont divisées en trois bases : «individu», «entreprise» et «non emploi». L'information sur le fait d'avoir été en apprentissage est issue de la base «individu», qui répertorie leurs caractéristiques individuelles, dont la formation qu'ils ont suivie. Dans la base «entreprise», une question renseigne sur le contrat de travail à l'embauche. Parmi les différents contrats de travail, on trouve le contrat d'apprentissage. Cependant, il est principalement collecté comme formation initiale, peu de situations étant renseignées dans la base «entreprise». Nous ne les retenons pas dans cette étude. Ainsi, sont comptabilisés ici comme apprentis les jeunes qui préparaient un diplôme en 2001 par l'apprentissage. Afin d'apprécier l'efficacité de l'apprentissage dans le supérieur au regard du secondaire, deux évaluations sont menées conjointement : l'une vise à comparer les jeunes qui ont effectué leur dernière année d'études supérieures en apprentissage et les jeunes ayant eu un diplôme de l'enseignement supérieur sans apprentissage; tandis que l'autre porte sur la comparaison des apprentis sortant du secondaire professionnel (CAP, BEP et Baccalauréat professionnel) avec les non-apprentis de ces mêmes filières. Nous excluons de notre évaluation les individus qui ont bénéficié d'un autre dispositif public afin d'isoler l'effet spécifique à l'apprentissage. Chez les sortants du supérieur, nous excluons également les diplômés des spécialités santé/social qui passent uniquement par des stages. Chez les sortants $\mathrm{du}$ secondaire, nous nous focalisons essentiellement sur le secondaire professionnel, autrement dit les diplômés d'un CAP, BEP ou Baccalauréat 
professionnel, qu'ils soient de spécialité tertiaire, industrielle ou comptabilité, car eux seuls peuvent bénéficier d'une formation par apprentissage.

\section{Le profil des bénéficiaires de l'apprentissage}

Le tableau 1 présente, pour le secondaire professionnel et pour le supérieur, les caractéristiques individuelles des apprentis comparativement à l'ensemble des sortants de formation initiale, ainsi que les résultats d'une analyse toutes choses égales par ailleurs. L'apprentissage n'a pas le même visage «en bas» et «en haut». Traditionnellement présents dans les spécialités industrielles, les apprentis du secondaire sont nombreux à effectuer leur apprentissage au sein d'un CAP industriel. Ces derniers représentent $14 \%$ de l'ensemble des effectifs du secondaire professionnel, mais près de $30 \%$ de ses apprentis. Cette forte différence est confirmée par une analyse toutes choses égales par ailleurs. Dans le supérieur, on retrouve cette place importante de l'apprentissage dans les diplômes de spécialité industrielle $(20 \%$ des apprentis du supérieur sont en BTS industriel). Mais ils sont également significativement plus présents dans les spécialités du tertiaire (avec également $20 \%$ des apprentis). Si l'on regroupe toutes les filières tertiaires de l'enseignement supérieur court, on atteint $41 \%$ des apprentis du supérieur. Une spécificité du supérieur tient au fait que l'apprentissage se développe dans les filières générales, notamment en Master 2. Les écoles de commerce et d'ingénieur ont également favorisé l'apprentissage dans leurs formations.

Concernant les caractéristiques individuelles, les apprentis du supérieur se différencient de ceux $\mathrm{du}$ secondaire. Dans le secondaire, les hommes sont sur-représentés chez les apprentis, que ce soit toutes choses inégales réunies ou égales par ailleurs, alors qu'aucune différence significative n'apparaît dans le supérieur. On aurait pu s'attendre à ce que les jeunes soient plus présents dans les filières en apprentissage lorsque leur père est artisan, commerçant ou chef d'entreprise. C'est plus souvent le cas pour les apprentis «d'en bas», mais cet effet n'apparaît pas significatif statistiquement. Cette variable semble moins influer sur ceux qui effectuent leur apprentissage dans l'enseignement supérieur, qui sont davantage issus d'un milieu favorisé que l'ensemble des jeunes sortis de formation initiale. On peut toutefois noter que les apprentis du secondaire sont plus nombreux à avoir un père employé ou ouvrier que les apprentis du supérieur (58\% contre $45 \%$ ). Alors que les apprentis ne se différencient pas de l'ensemble, que ce soit dans le supérieur ou dans le secondaire, en fonction de la situation du père (en emploi ou non), les apprentis du supérieur ont plus souvent une mère en emploi, toutes choses égales par ailleurs. Enfin, les jeunes d'origine étrangère sont moins présents au sein des formations en apprentissage, et cela plus particulièrement pour les apprentis du secondaire. Seuls $16 \%$ des apprentis ont un père né à l'étranger tandis qu'ils sont $21 \%$ au sein de l'ensemble des formations du secondaire.

Il existe de fortes inégalités régionales en matière d'apprentissage. Les régions prennent en charge une grande partie du financement de l'apprentissage et celui-ci représente désormais la moitié de leur dépense (MAINAUD, 2007). La région de formation est un facteur important en ce qui concerne le fait d'être apprenti ou pas. Sur nos données, on constate que l'Île-de-France concentre $27 \%$ des apprentis issus de l'enseignement supérieur, alors que cette même région rassemble $20 \%$ de l'ensemble des jeunes sortants de l'enseignement supérieur en 2001. Au contraire, le Nord, à l'instar d'autres régions, concentre $9 \%$ des sortants de l'enseignement supérieur mais ne forme que $5 \%$ des apprentis pour ces mêmes niveaux de formation. Ces résultats sont confirmés par l'estimation toutes choses égales par ailleurs ( $c f$. tableau 1). La forte territorialisation du contrat d'apprentissage s'explique par les lois de décentralisation qui ont conféré aux régions la gestion de l'apprentissage. Selon ARRIGHI et BROCHIER (2005b), la probabilité pour un jeune de 16 à 25 ans d'effectuer sa formation en apprentissage peut varier du simple au double selon les régions. L'analyse des statistiques descriptives nous montre que l'apprentissage est davantage présent dans certaines régions comme le Centre, le PoitouCharentes, l'Île-de-France ou les Pays de la Loire. Une autre disparité régionale réside dans la croissance de l'apprentissage dans les formations issues de l'enseignement supérieur. Certaines régions offrent davantage des formations en apprentissage dans le supérieur, tandis que pour d'autres, l'apprentissage reste ancré dans les formations du secondaire. De plus, le financement de l'apprentissage par la taxe d'apprentissage conduit à favoriser les grandes agglomérations qui accueillent sur leur territoire de grandes entreprises. Les politiques régionales en matière d'apprentissage, ainsi que les budgets associés, accentuent les disparités. 
Tableau 1 : Les caractéristiques individuelles des apprentis selon leur niveau de formation (en \%)

\begin{tabular}{|c|c|c|c|c|c|c|}
\hline & \multicolumn{3}{|c|}{ Secondaire } & \multicolumn{3}{|c|}{ Supérieur } \\
\hline & Apprentis & Coefficients & Ensemble & Apprentis & Coefficients & Ensemble \\
\hline \multicolumn{7}{|l|}{ Sexe } \\
\hline Homme & 77 & Réf. & 64 & 54 & Réf. & 46 \\
\hline Femme & 23 & --- & 36 & 46 & ns & 54 \\
\hline Âge & & +++ & & & +++ & \\
\hline$\hat{A} \mathbf{e}^{2}$ & & --- & & & --- & \\
\hline \multicolumn{7}{|l|}{ Diplômes et spécialités } \\
\hline $1^{\text {re }}$ année $C A P / B E P$ & 12 & Réf. & 12 & & & \\
\hline CAP/BEP industriel non diplômé & 16 & +++ & 13 & & & \\
\hline CAP industriel diplômé & 29 & +++ & 14 & & & \\
\hline BEP industriel diplômé & 7 & -- & 9 & & & \\
\hline CAP/BEP industriel comptabilité & 0 & --- & 0 & & & \\
\hline BAC pro industriel non diplômé & 3 & - & 3 & & & \\
\hline BAC pro industriel diplômé & 11 & -- & 11 & & & \\
\hline CAP/BEP tertiaire non diplômé & 5 & ns & 8 & & & \\
\hline CAP tertiaire diplômé & 6 & --- & 7 & & & \\
\hline BEP tertiaire diplômé & 4 & --- & 11 & & & \\
\hline CAP/BEP tertiaire comptabilité & 0 & --- & 1 & & & \\
\hline BAC pro tertiaire non diplômé & 1 & -- & 2 & & & \\
\hline BAC pro tertiaire diplômé & 6 & -- & 9 & & & \\
\hline Deug & & & & 4 & Réf. & 23 \\
\hline BTS/DUT industriel non diplômé & & & & 7 & +++ & 3 \\
\hline BTS industriel & & & & 20 & +++ & 9 \\
\hline DUT industriel & & & & 1 & ns & 2 \\
\hline BTS/DUT tertiaire non diplômé & & & & 15 & +++ & 6 \\
\hline BTS tertiaire & & & & 20 & +++ & 14 \\
\hline DUT tertiaire & & & & 5 & +++ & 3 \\
\hline $\mathrm{BAC}+3 \mathrm{LSH}$ & & & & 0 & --- & 7 \\
\hline $\mathrm{BAC}+4 \mathrm{LSH}$ & & & & 8 & +++ & 7 \\
\hline $\mathrm{BAC}+5 \mathrm{LSH}$ & & & & 5 & +++ & 7 \\
\hline Écoles de commerce & & & & 1 & --- & 3 \\
\hline $\mathrm{BAC}+3$ Maths & & & & 0 & --- & 1 \\
\hline BAC+ 4 Maths & & & & 6 & +++ & 2 \\
\hline BAC +5 Maths & & & & 1 & --- & 7 \\
\hline Écoles d’ingénieur & & & & 7 & +++ & 6 \\
\hline \multicolumn{7}{|l|}{ Lieu de naissance du père } \\
\hline France & 84 & Réf. & 79 & 86 & Réf. & 83 \\
\hline Étranger & 16 & --- & 21 & 14 & ns & 17 \\
\hline \multicolumn{7}{|l|}{ Enfants } \\
\hline Aucun & 92 & Réf. & 91 & 91 & Réf. & 91 \\
\hline Femmes avec enfants & 5 & -- & 7 & 6 & ns & 6 \\
\hline Homme avec enfants & 3 & ns & 2 & 3 & ns & 3 \\
\hline \multicolumn{7}{|l|}{ CSP du père } \\
\hline Employé/Ouvrier & 58 & Réf. & 60 & 45 & Réf. & 39 \\
\hline Agriculteurs, artisans, chefs d'entreprises & 18 & ns & 15 & 15 & ns & 16 \\
\hline Cadres, PI supérieures, techniciens & 13 & +++ & 13 & 40 & ns & 40 \\
\hline Père absent & 11 & ns & 12 & 9 & - & 5 \\
\hline \multicolumn{7}{|l|}{ Situation du père } \\
\hline En emploi & 96 & Réf. & 95 & 94 & Réf. & 90 \\
\hline Père au chômage & 4 & ns & 5 & 6 & ns & 10 \\
\hline
\end{tabular}




\begin{tabular}{|c|c|c|c|c|c|c|}
\hline & \multicolumn{3}{|c|}{ Secondaire } & \multicolumn{3}{|c|}{ Supérieur } \\
\hline & Apprentis & Coefficients & Ensemble & Apprentis & Coefficients & Ensemble \\
\hline \multicolumn{7}{|l|}{ Situation de la mère } \\
\hline En emploi & 73 & Réf. & 66 & 82 & Réf. & 74 \\
\hline Mère au chômage ou au foyer & 27 & ns & 34 & 18 & --- & 26 \\
\hline Île-de-France & 14 & Réf. & 14 & 27 & Réf. & 20 \\
\hline Picardie, Normandie, Bourgogne, Centre & 23 & ns & 21 & 18 & - & 17 \\
\hline Nord-Pas-de-Calais & 3 & --- & 8 & 5 & - & 9 \\
\hline Lorraine, Alsace, Franche-Comté & 11 & $\mathrm{~ns}$ & 12 & 10 & - & 9 \\
\hline Bretagne, Pays de la Loire, Poitou-Charentes & 17 & ns & 14 & 14 & --- & 16 \\
\hline Aquitaine, Limousin, Midi-Pyrénées & 11 & ns & 10 & 7 & --- & 8 \\
\hline Rhône-Alpes, Auvergne & 12 & ns & 12 & 14 & - & 13 \\
\hline $\begin{array}{l}\text { Languedoc, Provence-Alpes-Côte d'Azur, } \\
\text { Corse }\end{array}$ & 9 & - & 9 & 5 & --- & 8 \\
\hline
\end{tabular}

Note : Dans la colonne «coefficients» est reporté le signe du coefficient (en fonction de sa significativité) issu de l'estimation de la probabilité d'être passé par l'apprentissage en fonction des différentes variables. +++ indique un coefficient positif et significatif au seuil de $1 \% ;++$ indique un coefficient positif et significatif au seuil de $5 \%$; + indique un coefficient positif et significatif au seuil de $10 \%$. Idem pour ---, -- et - .

Champ : Ensemble des jeunes sortis de l'enseignement secondaire professionnel et supérieur en 2001.

Source : Enquête «Génération» 2001-Interrogation 2004, Céreq.

\section{La méthode d'estimation}

Notre analyse se veut multidimensionnelle afin de mesurer l'insertion sur le marché du travail des jeunes passés par l'apprentissage. Nous retenons deux critères : avoir occupé un emploi d'au moins six mois un an après la sortie de formation initiale et le salaire alors perçu dans cet emploi. Afin d'évaluer empiriquement l'effet de l'apprentissage, nous comparons les résultats de différentes estimations (cf. tableau 2).

\section{Évaluer l'accès à l'emploi}

L'influence de l'apprentissage sur la probabilité d'obtenir un emploi est tout d'abord estimée dans un modèle probit simple sans correction particulière autre que de tenir compte d'un ensemble de variables de contrôle (modèle 1). On évalue l'impact d'être passé par l'apprentissage sur la probabilité d'être en emploi (de plus de six mois) un an après, toutes choses égales par ailleurs. Les variables de contrôle utilisées sont le sexe, l'âge, l'origine sociale (CSP $\mathrm{du}$ père, situation professionnelle du père et de la mère), le lieu de naissance du père, les niveaux et les spécialités de formation, le fait d'avoir des enfants, et enfin les indicatrices de régions.

Cependant, comme nous l'avons vu précédemment, au-delà des caractéristiques observables dont on peut tenir compte, les bénéficiaires de l'apprentissage peuvent avoir des caractéristiques spécifiques inobservées relativement aux non bénéficiaires. Autrement dit, même après avoir pris en compte des variables de contrôle décrites ci-dessus, les apprentis peuvent ne pas être répartis de manière aléatoire dans la population des sortants de formation initiale. Si tel est le cas, cela biaise l'évaluation de l'effet de l'apprentissage sur le fait d'être en emploi ou sur le salaire. Si ces caractéristiques inobservées influencent à la fois le fait d'entrer en apprentissage (ou pas) et la variable de résultat, on parle d'effet de sélection. Cet effet est notamment testé dans les travaux de Simmonet et UlRich (2000). Par exemple, si les jeunes apprentis disposent ex ante d'un capital social plus élevé, ils auront certainement des chances différentes de trouver un emploi rapidement après leur formation et gagneront des salaires plus élevés (Sollogoub, Ulrich, 1999). Le rendement mesuré peut alors être la combinaison, non seulement de l'effet spécifique du fait d'effectuer sa formation en alternance, mais également de l'effet de sélection.

Afin de corriger de cet éventuel biais, on utilise un probit bivarié (modèle 2). L'intérêt de ce modèle est d'estimer conjointement la probabilité d'être passé par l'apprentissage et la probabilité d'obtenir un emploi. Pour permettre l'identification de l'effet de l'apprentissage, nous devons introduire une variable de type instrumental fortement corrélée avec le fait d'être passé par l'apprentissage mais qui n'influence pas directement la probabilité d'être en emploi. Cet instrument permet d'estimer l'effet spécifique de l'apprentissage. Cependant, le choix d'un instrument valide est toujours délicat, alors même que sa non-pertinence peut fragiliser les résultats. Ici, nous exploitons la même stratégie qu'Elodie ALET et Liliane Bonnal (2011) en introduisant le taux d'apprentissage par région au cours de l'année 2001 (année au cours de laquelle les jeunes effectuent leur apprentissage) comme premier instrument. En effet, étant donné la forte territorialisation du contrat d'apprentissage, la région de formation joue un rôle primordial dans le développement de ce dispositif. On peut alors supposer que la part d'apprentis au niveau régional influence la probabilité d'effectuer une formation par apprentissage mais pas directement la probabilité d'accès à l'emploi. Avant même la loi du 7 janvier 1983 qui confie à la région la gestion de l'apprentissage, l'apprentissage n'était 
déjà pas réparti de manière homogène sur le territoire français (ARRIGHI, BRochIER, 2005b). À ces inégalités déjà existantes, se sont ajoutées des politiques régionales diversifiées qui les ont accentuées. Afin de renforcer cet instrument, nous introduisons un effet croisé : la part d'apprentis par régions (pour le supérieur d'une part, et pour le secondaire professionnel d'autre part) croisée avec le type de filière suivie. L'idée sous-jacente à l'introduction de cet instrument vient du fait qu'en fonction du niveau d'apprentis formés dans une région, dans le supérieur ou dans le secondaire, l'effet sur la probabilité du jeune de préparer un diplôme par apprentissage sera différent selon la voie de formation suivie. Une région qui développe davantage l'apprentissage aura un impact différent sur le fait qu'un jeune devienne apprenti, selon qu'il suit ses études supérieures dans la voie universitaire ou bien au sein d'une grande école. Dans l'estimation pour les jeunes sortis diplômés de l'enseignement supérieur, nous croisons ainsi le taux d'apprentissage au niveau régional par le fait d'être dans une filière courte technologique (BTS et DUT), dans une filière universitaire, et dans une grande école (de commerce ou d'ingénieur). Dans l'estimation pour les jeunes sortants de l'enseignement secondaire professionnel, nous croisons le taux d'apprentissage au niveau régional par le fait de préparer un CAP, un BEP ou un baccalauréat professionnel. Les chances d'être en apprentissage durant sa dernière année de formation initiale dépendent fortement de la proportion d'apprentis dans la région de formation(10), ce qui n'aura pas le même effet selon la voie de formation suivie par le jeune.

\section{Évaluer les écarts de salaire}

L'effet de l'apprentissage sur le salaire est tout d'abord estimé sans correction particulière dans le modèle 3 par une équation de salaire qui tient compte d'un certain nombre de variables observables (individuelles et d'entreprise) ( $c f$. tableau 3). Dans le modèle 4, afin de mesurer les écarts de salaires entre ceux qui ont bénéficié de l'apprentissage et ceux qui sont passés par la voie classique, nous estimons une équation de salaire en tenant compte du biais lié aux caractéristiques spécifiques des apprentis qui rentrent dans une formation par apprentissage. Nous estimons l'équation de salaire sur la population des individus qui occupent un an après leur sortie de formation initiale un emploi de plus de six mois. L'effet de l'apprentissage sur le logarithme du salaire est estimé en introduisant la probabilité estimée de passer par l'apprentissage dans l'équation de salaire. On utilise

(10) Nous n'avons pas reporté ici les résultats de la première équation du probit bivarié (probabilité d'effectuer sa dernière année de formation initiale en apprentissage) pour le supérieur d'une part, et pour le secondaire professionnel d'autre part. Ces résultats sont disponibles auprès de l'auteur. Notons que la proportion d'apprentis au niveau régional est positive et fortement significative dans les deux cas. ici les mêmes variables explicatives que précédemment (caractéristiques individuelles), auxquelles on rajoute des variables d'entreprise comme la taille de l'entreprise et le secteur d'activité.

\section{L'impact de l'apprentissage sur la situation professionnelle future}

\section{Effet sur l'accès à l'emploi}

Afin d'évaluer l'effet d'avoir bénéficié de l'apprentissage sur l'accès à l'emploi un an après la sortie de la formation initiale (secondaire et supérieur), nous comparons les résultats des deux estimations menées : un probit simple (modèle 1) puis un probit bivarié (modèle 2).

Les résultats obtenus pour le secondaire confirment ceux décrits dans les études antérieures (cf. supra). Ils montrent ainsi que l'apprentissage favorise l'accès à un emploi d'au moins six mois, avec ou sans correction. L'effet est positif et significatif. L'obtention du diplôme à la fin de la formation initiale protège toujours du chômage. L'âge est également un facteur qui favorise la probabilité d'être en emploi. Le fait d'avoir des enfants a un impact négatif et significatif pour les femmes, tandis qu'il est non significatif pour les hommes.

Pour le supérieur, les résultats de l'estimation du modèle 1 montrent que l'apprentissage favorise la probabilité d'obtenir un emploi un an après être sorti de formation toutes choses égales par ailleurs, et cela de manière significative. Cependant, quand on tient compte du caractère non aléatoire de l'apprentissage, l'impact de l'apprentissage devient non significatif (modèle 2). Ainsi, lorsque l'on neutralise l'effet des caractéristiques spécifiques de ceux qui rentrent en apprentissage (davantage que par un simple contrôle des variables observables), avoir été apprenti du supérieur ne permet pas d'accéder plus facilement à un emploi un an plus tard comparativement à ceux qui sont passés par la voie scolaire, toutes choses égales par ailleurs. Ainsi, ces résultats indiquent que les apprentis du supérieur ne bénéficient pas d'une meilleure insertion professionnelle car ils sont passés par une formation en apprentissage, mais parce qu'ils ont des caractéristiques particulières qui expliqueraient qu'ils sont plus souvent en emploi. Ces deux estimations confirment l'influence d'un certain nombre d'autres variables. Les caractéristiques individuelles des diplômés du supérieur restent en effet essentielles pour expliquer l'insertion sur le marché du travail (SANCHEZ, 2004). C'est le cas du diplôme : les titulaires d'un troisième cycle en sciences et mathématiques ou d'une école d'ingénieur ont une probabilité plus grande d'accéder à un emploi un an après être sorti de formation initiale. C'est également le cas des BTS. 
Tableau 2 : Probabilité d'être en emploi un an après la sortie du système de formation initiale

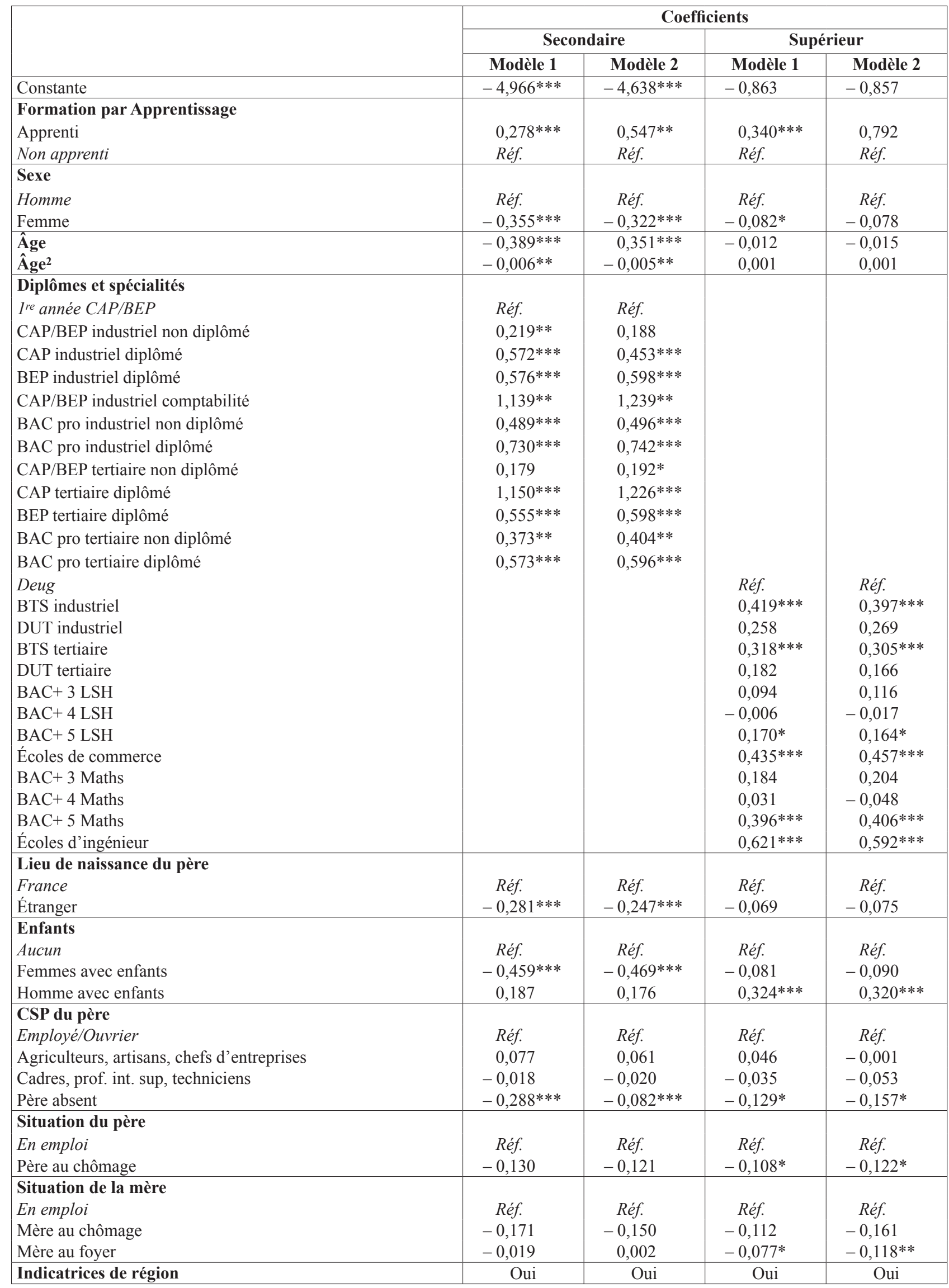

Niveau de significativité : ${ }^{* * *}$ significatif au seuil de $1 \%$; ** significatif au seuil de $5 \%$; * significatif au seuil de $10 \%$. Champ : Ensemble des jeunes sortis de l'enseignement secondaire professionnel et supérieur en 2001.

Source : Enquête «Génération» 2001-Interrogation 2004, Céreq. 
Tableau 3 : Équation de salaire

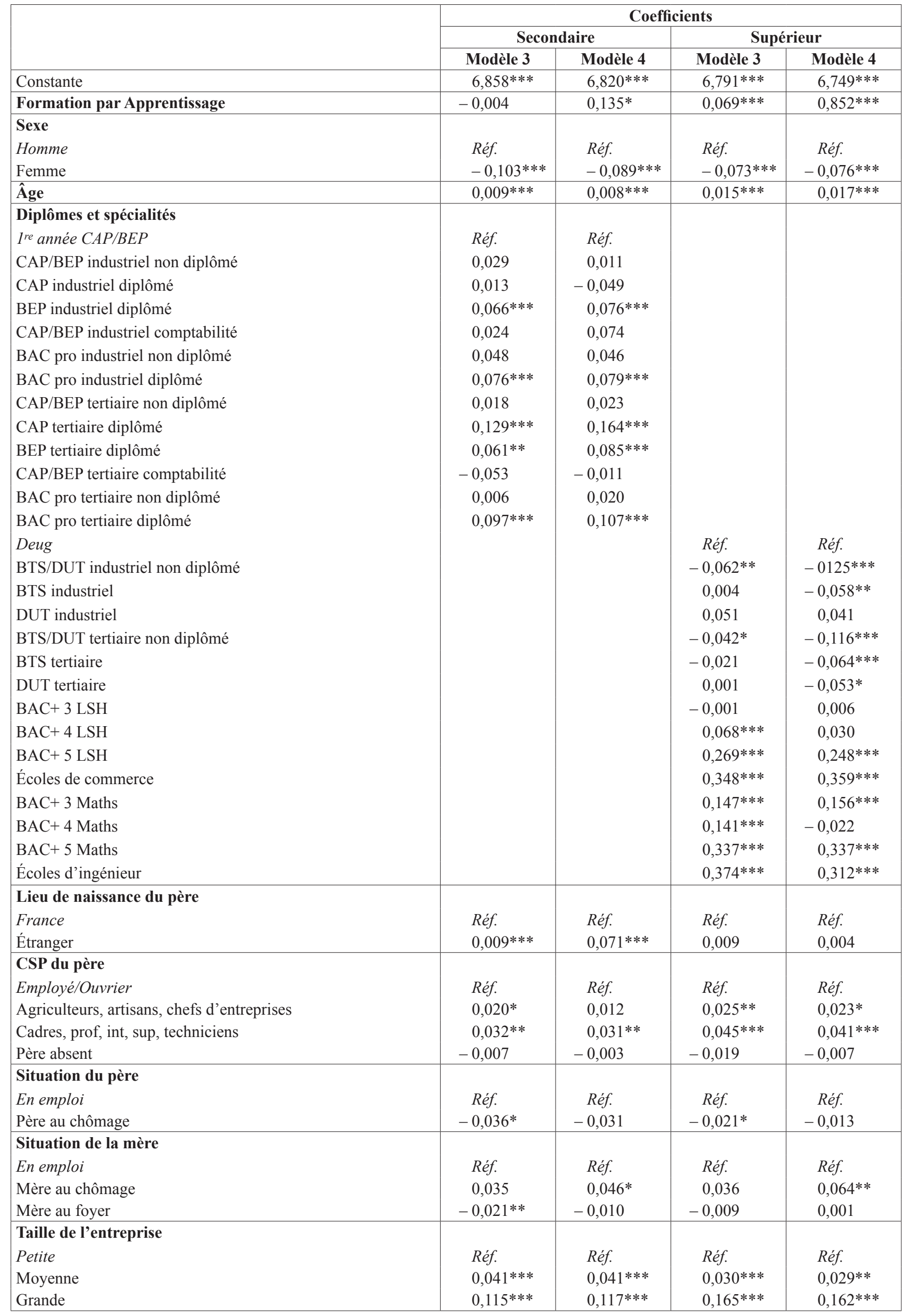




\begin{tabular}{|l|c|c|c|c|}
\hline \multirow{2}{*}{} & \multicolumn{3}{|c|}{ Coefficients } \\
\cline { 2 - 5 } & \multicolumn{2}{|c|}{ Secondaire } & \multicolumn{2}{c|}{ Supérieur } \\
\cline { 2 - 5 } Secteurs & Modèle 3 & Modèle 4 & Modèle 3 & Modèle 4 \\
Culture/ éducation/santé & & & & \\
Industrie & Réf. & Réf. & Réf. & Réf. \\
Finance, ingénierie, conseil, info & $-0,063^{* * *}$ & $-0,063^{* * *}$ & $0,031^{* *}$ & $0,031^{* *}$ \\
Commerce, services & $-0,105^{* * *}$ & $-0,020$ & $-0,027^{* *}$ & $0,052^{* * *}$ \\
Hôtels, restaurants, transports & $-0,019$ & $-0,106^{* * *}$ & $0,054^{* * *}$ & $-0,025^{*}$ \\
Agriculture & $-0,064^{* * *}$ & $-0,065^{* * *}$ & $-0,019$ & $-0,021$ \\
\hline Temps de travail & $-0,112^{* * *}$ & $-0,114^{* * *}$ & $-0,090$ & $-0,070$ \\
Temps plein & & & & \\
Temps partiel & Réf. & Réf. & Réf. & Réf. \\
\hline
\end{tabular}

Niveau de significativité : ${ }^{* * *}$ significatif au seuil de $1 \%$; ** significatif au seuil de $5 \%$; ${ }^{*}$ significatif au seuil de $10 \%$.

Champ : Ensemble des jeunes sortis de l'enseignement secondaire professionnel et supérieur en 2001

Source : Enquête «Génération» 2001-Interrogation 2004, Céreq.

\section{Effet sur le salaire}

Dans le secondaire, l'effet sur le salaire est très faiblement négatif et non significatif dans le modèle 3 et devient positif mais faiblement significatif dans le modèle 4. Ce faible impact sur le salaire perçu un an après la sortie de formation initiale effectuée en apprentissage montre que l'apprentissage pour les sortants du secondaire professionnel n'améliore pas de façon significative leurs salaires. Sortir diplômés de BEP ou de baccalauréat professionnel améliorent en revanche significativement le salaire alors perçu. L'effet de l'apprentissage sur le salaire est beaucoup plus important dans le supérieur.

Les résultats de l'estimation du modèle 3 , confirmés dans le modèle 4 , montrent que le fait d'avoir bénéficié d'une formation en apprentissage dans l'enseignement supérieur a un effet positif sur les salaires des personnes en emploi un an après être sorti de la formation initiale, et cela de manière significative. Autrement dit, effectuer sa dernière année dans l'enseignement supérieur en apprentissage permet, toutes choses égales par ailleurs, d'obtenir un salaire plus élevé un an après comparativement aux non apprentis. L'estimation tient compte des caractéristiques spécifiques des apprentis, plus précisément nous introduisons dans notre modèle la probabilité d'avoir effectué sa dernière année dans l'enseignement supérieur en apprentissage (11). Ce résultat peut s'expliquer par l'ancienneté acquise dans l'entreprise si le jeune continue à travailler dans le même établissement que celui où il a effectué sa formation en apprentissage, soit par l'expérience acquise qui l'amène à accéder à un salaire supérieur à ceux qui n'ont pas bénéficié de cette expérience. Ces «faux débutants» issus de l'enseignement supérieur bénéficieraient alors d'une plus-value en terme salarial sur le marché du travail. D'autres variables

(11) Le fait de corriger du caractère non aléatoire de l'apprentissage n'a pas permis de corriger également d'un éventuel biais de sélection lié au fait de mener l'estimation de l'équation de salaire uniquement sur les personnes en emploi. ont également une influence sur les salaires perçus. Le fait d'être une femme diminue significativement le salaire relativement aux hommes, ce qui confirme l'existence d'inégalités salariales de sexe, toutes choses égales par ailleurs. Le niveau et la spécialité du diplôme jouent un rôle déterminant. Plus l'individu est diplômé, plus sa probabilité d'accéder à des postes rémunérateurs est grande : le niveau de formation reste un facteur important quant à l'existence d'un gain net en salaire. Les filières scientifiques offrent de meilleurs salaires a posteriori que les filières de lettres et sciences humaines. L'origine sociale influence significativement le niveau du salaire : les enfants de cadres et de chefs d'entreprises ont plus de chances d'accéder à des emplois plus rémunérateurs que les enfants d'employés et d'ouvriers.

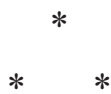

L'alternance connaît une importance croissante et cette évolution s'accompagne d'une augmentation des niveaux de diplômes des jeunes bénéficiaires. Les contrats en alternance concernent de plus en plus des jeunes diplômés du supérieur, alors que ces dispositifs ont été initialement mis en place pour pallier une formation insuffisante ou inadéquate des jeunes les plus touchés par le chômage. Il semble donc que les changements récents tendent à éloigner l'alternance de son objectif premier.

Les résultats de nos estimations confirment ceux d'études antérieures, indiquant que, pour les sortants $\mathrm{du}$ secondaire professionnel, l'apprentissage permet d'améliorer les chances d'accéder à un emploi, mais avec un effet faible sur les salaires alors perçus. En revanche, les résultats permettent de montrer que si, pour les sortants de l'enseignement supérieur, l'apprentissage a un impact positif sur la probabilité d'être en emploi un an après être sorti de formation initiale, cet impact devient non significatif quand on tient compte des caractéristiques spécifiques des 
apprentis. Ce ne serait donc pas l'apprentissage qui les conduirait à être plus souvent en emploi mais leurs caractéristiques propres. Le facteur le plus discriminant reste le niveau de diplôme et la spécialité de formation. Par ailleurs, le fait d'être passé par l'apprentissage a une influence positive sur les salaires des jeunes en emploi un an après être sorti de leur formation.

Notre étude se limite à estimer l'efficacité de l'apprentissage à partir de deux indicateurs seulement : l'emploi et le salaire un an après. L'exploitation des données plus récentes, où l'échantillon des sortants du supérieur passés par l'apprentissage est plus important, nous permettra de prolonger ce travail. L'utilité du dispositif pour les moins diplômés n'est pas remise en cause ici, mais nos résultats conduisent à s'interroger sur le fait de l'étendre aux plus diplômés. Dans un contexte où le taux de chômage touche majoritairement les moins diplômés, on peut conclure que l'apprentissage a tout son intérêt dans le secondaire, mais l'on peut s'interroger sur l'utilité de financer ce type de formation pour des jeunes plus diplômés qui sont déjà favorisés en termes d'insertion. Les pouvoirs publics prennent en charge une partie du coût du travail des apprentis par le biais d'exonérations sociales et d'aide à l'embauche dans le but de faciliter le recrutement de ces jeunes par le biais de ces contrats. On peut se questionner sur l'existence d'effets d'aubaine pour les employeurs quant à l'utilisation de ces contrats. L'évaluation des dispositifs en alternance gagnerait notamment à être approfondie par une étude des comportements des employeurs quant à l'utilisation de ces contrats.

\section{Bibliographie}

Abriac D., Rathelot R., SAnchez R. (2009), «L'apprentissage entre formation et insertion professionnelles », Formations et Emploi. Édition 2009, INSEE.

Acemoglu D., Pischke J.-S. (1998), "Why do firms train? Theory and evidence", Quarterly Journal of Economomics 1113, pp. 79-119.

Acemoglu D., Pischke J.-S. (1999), "Beyond Becker: training in imperfect labour markets", the Economic Journal, vol. 109, № 453, F112-F142.

Alet E., Bonnal L. (2011), "Vocational schooling and educations success: comparing apprenticeship to fulltime vocational high school", document de travail, Laboratoire CRIEF-TEIR, T2011-02.

Arrighi J.-J., Brochier D. (2005a), «1995-2003, l'apprentissage aspiré par le haut», Bref, n 217, Céreq, mars.

Arrighi J.-J., BRochiER D. (2005b), «L'apprentissage au sein de l'Éducation nationale : un état des lieux», rapport du haut Conseil de l'évaluation à l'École, no 17, avril.

ARrighi J.-J., JosEPH O. (2005), «L'apprentissage : une idée simple, des réalités diverses », Céreq, Bref, no 23, octobre.

Bender S., Schwerdt W. (2000), How many apprentices? A comparaison of firms 'training behaviour in France and Germany, Mimeo, CREST-INSEE.

Bonnal L., Fougère D., Serandon A. (1997), “Evaluating the Impact of French Employment Policies on Individual Labour Market Histories", Review of economic studies, vol 64 (4), October, pp. 683-713.

Brodaty T., Crépon B., Fougère D. (2007), «Les méthodes microéconométriques d'évolution et leurs applications aux politiques actives de l'emploi», Économie et Prévision, no 177.
Durier S., SAING P. (2007), «Le développement de l'apprentissage depuis les années quatre-vingt-dix», no 75 , Education et Formation, octobre.

Even K., KleIN T. (2006), «La politique spécifique de l'emploi et la formation professionnelle : un profit à moyen terme pour les participants?», Document d'étude, DARES.

Fougère D., Schwerdt W. (2002), "Are Apprentices Productive?" Applied Economics Quarterly, 48, pp. 317-346.

Fougère D., Kramarz F., Magnac T. (2000), "Youth Employment Policies in France", European Economic Review, 44, pp. 928-942.

Fougere D., Goux D., Maurin E. (2001), «Formation continue et carrières salariales : une évaluation sur données individuelles», Annales d'Économie et Statistique 62, pp. 49-70.

Giret J.-F., Molinari-Perrier M., Moulle S. (2006), «2001-2004 : les sortants de l'enseignement supérieur face au marché du travail», NEF nº 21, Céreq, mars.

Hetzel P. (dir.) (2006), Del'Université à l'Emploi, rapport final, Commission du débat national Université-Emploi.

KeRgOAT P. (2010), «Les formations par apprentissage : un outil au service d'une démocratisation de l'enseignement supérieur», Net. Doc, CEREQ, no 75, décembre.

LindLEY R.M. (1975), "The demand for apprentice recruits by the engineering industry, 1951-1971", Scottish Journal of Political Economy 22, pp. 1-24.

Lunel P. (dir.) (2007), Pour un nouveau pacte avec la jeunesse, Schéma national de l'orientation et de l'insertion professionnelle. 
Mainaud T. (2007), «La dépense nationale pour la formation professionnelle continue et l'apprentissage en 2005 », Premières Synthèses, DARES, no 45.3.

SANCHEZ R. (2004), «Les contrats d'apprentissage et de qualification : les caractéristiques individuelles des bénéficiaires restent essentielles pour expliquer l'insertion ", Document d'étude, DARES, nº 05.1, janvier.

SANCHEZ R. (2010), «L'apprentissage en 2008 : hausse des entrées et du niveau de diplôme préparé», DARES Analyses n 024, avril.
SANCHEZ R. (2008), «L'apprentissage en 2006 : les entrées s'accroissent surtout dans le tertiaire», Premières Synthèses, DARES, no 15.1, avril.

Simmonet V., Ulrich V. (2000), «La formation professionnelle et l'insertion sur le marché du travail : l'efficacité du contrat d'apprentissage», Économie et Statistique, no 337-338.

Sollogoub M., Ulrich V. (1999), «Les jeunes en apprentissage ou en lycée professionnel, une mesure quantitative et qualitative de leur insertion sur le marché du travail», Économie et Statistique, no 323, pp. 31-52. 